\title{
La regulación jurídica de los partidos políticos en México desde la constitucionalización semántica de los partidos de 1963 hasta la elección presidencial de 1976: un ejercicio de retrospectiva histórica comparada con énfasis especial en el voto de los jóvenes
}

The Legal Framework Of Political Parties in Mexico from the Semantic Constitutionalization of the Parties from 1963 to the Presidential Election of 1976: a Comparative Retrospective Study with an Emphasis on the Youth Vote

\section{Francisco J. de Andrea Sánchez*}

\section{Sumario:}

I. La constitucionalización semántica de los partidos políticos de 1963

II. Las reformas de 1963 a la Ley Federal Electoral de 1951

III. Las reformas electorales de 1970 en México

IV. Las reformas constitucionales de 1972 al sistema electoral

V. La Ley Federal Electoral de 1973

* Investigador en el Instituto de Investigaciones Jurídicas de la UNAM. 


\section{Resumen:}

El objetivo de este artículo consiste en lograr una revaloración del proceso gradual de reformas político-electorales en materia de partidos políticos y del voto de los jóvenes desde 1963 hasta 1976, que permita a las generaciones actuales de jóvenes equilibrar su ímpetu transformador de instituciones político-electorales con una perspectiva histórico comparativa que retrospectivamente permite aquilatar los logros, el progreso gradual y la visión de un buen número de mexicanos visionarios, que ya sea en su función de investigadores, de profesores, de legisladores o de funcionarios públicos contribuyeron a construir un andamiaje jurídico constitucional electoral con muchos episodios brillantes y algunas regresiones, pero que en balance nos coloca favorablemente en el espectro institucional organizativo y administrativo de los procesos electorales modernos y que por lo tanto deben evitarse los dos extremos de un debate falso que busca irreflexivamente dinamitar el territorio teórico y práctico ya conquistado, o bien regresar a un arcaísmo político electoral también ya superado.

\begin{abstract}
:
This essay seeks to shed light on the positive nature of the gradual amendment process in the realm of the legal framework of political parties in Mexico from 1963 to 1976 which will in turn allow new generations of Mexican firsttime voters in 2012 to value the progress and advances achieved by outstanding Mexican academicians, lawyers, legislators and politicians who collectively and in the last 50 years have managed to improve an electoral system which in many areas is a state of the art legal construction and thus avoid he extremes of a false debate which posits either abandoning a workable and sophisticated system or a return of sorts to an archaic and obsolete electoral system.
\end{abstract}

Palabras clave: partidos políticos, reforma electoral, sistema electoral, voto joven

Descriptors: political parties, electoral reform, electoral system, youth vote 


\section{La constitucionalización semántica de los partidos políticos de 1963}

Este ensayo constituye un ejercicio comparativo histórico-retrospectivo que nos ayudará a resaltar dramáticamente lo mucho que ha avanzado México en la regulación jurídica de los partidos políticos en las últimas cinco décadas - progreso gradual e innegable que algunos ignoran y otros olvidan - y nos sirve como un recurso de emergencia para concientizarnos -en vísperas de la elección Presidencial de 2012 - sobre los costos que dicha evolución gradual ha tenido para miles de ciudadanos, intelectuales, periodistas y políticos mexicanos, que incluso han dado hasta la vida en la construcción de un andamiaje jurídico que en la actualidad corre el riesgo de caer -injustamente como veremos - en el descrédito mayoritario de no corregirse, por citar tan solo dos ejemplos, las desviaciones y patologías evidentes en la constitución y funcionamiento de algunos partidos políticos "fachada" o "familiares" que desvirtúan grotescamente el espíritu progresista del largo camino legislativo que ha recorrido México en materia de la regulación jurídica de los partidos políticos y de algunos otros aspectos esenciales del sistema electoral vigente que en su momento significaron avances vanguardistas de la legislación comicial mexicana como el del modelo de asignación de tiempos y espacios disponibles en los medios masivos de comunicación electrónicos para la programación de contenido político electoral.

La reforma constitucional del 22 de julio de 1963, constitucionalizó a los partidos políticos en México, al incorporar a la ley fundamental de 1917 la figura de los llamados "diputados de partido". La citada reforma, según nuestro punto de vista, configuró un nivel de constitucionalización —el semántico-, aunque no se desglosara el concepto y las funciones esenciales de los partidos, como sucedió - por ejemplo- con las Constituciones europeas de la segunda posguerra y posteriormente en nuestro propio país con las reformas de 1977. De esta forma, el constitucionalismo mexicano puede ufanarse de haber "constitucionalizado" formalmente a los partidos desde 1963, debido a que no sólo la introducción integral de la temática partidista en un texto fundamental constituye una "constitucionalización"; puesto que también una simple mención semántica de los partidos - como la insertada por la enmienda de 1963 a nuestra carta magna - da lugar a 
poder hablar de una constitucionalización de los partidos políticos, aunque tan solo sea de nivel semántico.

A mayor abundamiento, desde la anterior perspectiva, la constitucionalización semántica implica un reconocimiento por parte del Estado mexicano de la importancia creciente e innegable de los partidos políticos en el funcionamiento no sólo de los sistemas políticos sino de las sociedades en general. Asimismo, bajo un análisis profundo de las dinámicas de la psicología del poder que operaron en la génesis de la creación de los llamados diputados de partido en México, está el hecho de que desde la sociedad comenzaba a surgir una demanda creciente de que el Estado mexicano se inclinara a reconocer la importancia sistémica e institucional de los partidos políticos en la intermediación política entre el Estado y los ciudadanos y en la "domesticación" y canalización pacífica de los conflictos sociales. Ante esta demanda, el Estado - vía la constitucionalización semántica- y en un ejercicio de ingeniería legislativa preventiva calculó que era tiempo de fomentar así fuese incipientemente y a través de un reconocimiento inicialmente semántico el papel crucial que desempeñarían los partidos políticos en el sistema electoral mexicano a mediano y largo plazo como válvula de escape institucional para canalizar inquietudes que de otra forma podrían expresarse en la marginalidad o clandestinidad. Esta postura "preventiva" del legislador mexicano alcanzaría un nivel climático con el reconocimiento y constitucionalización integrales, ya no sólo semántico, de los partidos políticos con la reforma política de 1977.

Para comprender bien el porqué de la creación de la figura de los diputados de partido, a continuación recordaremos brevemente la situación política imperante en México en 1962.

Al comenzar la década de los sesenta, el panorama político mexicano dejaba ver un sistema de partidos en el que el Partido Revolucionario Institucional seguía firme con un poder hegemónico indisputado.

Por otro lado, la raquítica oposición "era pálida, desvertebrada, esporádica”. ${ }^{1}$ En 1962, el PAN y el PPS estaban exhaustos. El divisionismo interno de estos partidos debilitaba a la incipiente oposición. El PARM aparecía como un germen de partido, cuyos integrantes eran antiguos militares retirados. ${ }^{2}$

${ }^{1}$ Cfr. López Moreno, Javier, La reforma política en México, México, Centro de Documentación Política, 1979, p. 68.

2 Cfr. Paoli, Francisco, "Legislación electoral y proceso político", Jurídica, t. I, núm. 10, julio de 1978, p. 201. 
Para comprender mejor el panorama de crisis que presentaba el sistema de partidos en México, basta con examinar las estadísticas electorales que son elocuentes.

En las elecciones de 1955, el Partido Acción Nacional (PAN) obtuvo seis curules, el Partido Popular Socialista (PPS) dos y el Partido Auténtico de la Revolución Mexicana (PARM) ninguna.

En los comicios de 1958, el PAN obtuvo cinco curules, el PPS y el PARM una cada uno; en las de 1961, el PAN obtuvo cuatro, el PPS una y el PARM ninguna. El balance: en diez años toda la oposición obtuvo un total de 20 curules en la Cámara de Diputados, de un total de 483 que se disputaron en ese lapso. Por lo que a senadurías y gubernaturas respecta, la oposición se fue en blanco.

De esta forma, el 22 de julio de 1963, en el Diario Oficial de la Federación se publicaron sendas reformas y adiciones a los artículos 54 y 63 constitucionales. La principal innovación aportada por estas reformas fue la creación de los llamados diputados de partido. El régimen de los diputados de partido quedó asentado en el artículo 54 constitucional.

El objetivo profundo de la reforma al artículo 54 era apuntalar la estabilidad política que el país había logrado obtener. Porque, como estableció Miguel de la Madrid Hurtado, en su etapa de investigador del derecho constitucional, "las reformas mencionadas constituyen la medida más seria y trascendente que se ha adoptado en México en materia de instituciones políticas desde la consolidación de la prohibición constitucional absoluta para la reelección del presidente de la República". ${ }^{3}$

De esta manera, "la filosofía que animó la creación de los diputados de partido, fue alentar a la oposición, facilitarle su ingreso a la discusión en la Cámara de Diputados, dar cauce legítimo a los partidos minoritarios y preservar el sistema de gobierno mexicano".

Bajo el novedoso sistema de los diputados de partido se llevaron a cabo las elecciones de 1964, en las que el PAN obtuvo veinte curules, el PPS diez, y el PARM cinco; las de 1967 en las que el PAN obtuvo diecinueve, el PPS diez y el PARM cinco, y las de 1970, en las cuales el PAN obtuvo veinte curules, el PPS diez y el PARM cinco.

3 De la Madrid H., Miguel, "Reforma a la Constitución federal en materia de representación”, Revista de la Facultad de Derecho de México, México, núm. 50, 1963, p. 356.

4 Cfr. López Moreno, Javier, op. cit., p. 70. 


\section{Las reformas de 1963 \\ a la Ley Federal Electoral de 1951}

La Ley Electoral de 1951 fue reformada y adicionada mediante un decreto del 28 de diciembre de 1963, para ponerla a tono con las reformas constitucionales de los artículos 54 y 63. Este decreto también introdujo la figura de las exenciones fiscales para los partidos políticos legalmente registrados y estableció algunos ajustes para el caso de las confederaciones y coaliciones de partidos tratándose de los diputados de partido.

\section{Las exenciones fiscales}

Mediante el decreto del 28 de diciembre de 1963, se creó el artículo 44 bis, que estableció la exención - a favor de los partidos- de impuestos del timbre; los relacionados con rifas o sorteos y festivales; sobre la renta y los que se causen por la venta de los impresos de los partidos. La importancia de este artículo radica en que constituye la primera disposición electoral mexicana que establece un cierto tipo de ayuda económica, de financiamiento "primitivo" de los partidos políticos. "Esta conquista no tendría vuelta atrás", ${ }^{5}$ pues en las leyes posteriores fue ampliada gradualmente.

\section{La calificación de las elecciones}

Los artículos 127 y 134 de la Ley Electoral Federal también fueron modificados para dar cabida a los diputados de partido. Así, el texto del artículo 127 reformado estableció que:

La Cámara de Diputados calificará la elección de sus propios miembros. Su resolución será definitiva e inatacable.

I. En primer término, resolverá sobre la elección de los diputados que hubiesen obtenido mayoría de votos en su distrito;

II. Enseguida, efectuará el cómputo total de votos emitidos en la República para conocer de la elección de los diputados de partido;

\footnotetext{
5 Ibidem, p. 74.
} 
III. Con base en el Artículo 54 de la Constitución Política de la República, determinará el número de diputados de partido a que tenga derecho cada uno de los Partidos Políticos Nacionales y sin deducir los votos en los distritos donde hubieren alcanzado mayoría;

IV. A continuación, formulará una lista de los candidatos de cada partido que resultaren con derecho a ser diputados de partido, anotándose en riguroso orden, de acuerdo con el número decreciente de sufragios que hayan logrado en relación a los demás candidatos del mismo partido, en todo el país, y procederá a hacer la declaratoria respectiva;

V. Serán diputados de partido suplentes los que hayan figurado como suplentes de los respectivos candidatos propietarios que resulten electos.

Cabe destacar, respecto a este artículo, que la fracción IV reviste gran interés, porque para velar por la eficacia práctica del principio de la soberanía popular en el proceso electoral, y dejar la decisión definitiva al electorado, se fijó que la designación de los diputados de partido no sería dejada al arbitrio de las nomenclaturas de los partidos; sino que serían declarados electos, en orden de preferencia, los candidatos que, no habiendo alcanzado la mayoría, hubieran logrado el más alto porcentaje de sufragios en relación con otros integrantes del mismo partido.

La anterior disposición buscaba evitar la "oligarquización" de los partidos políticos, desgraciadamente, la buena intención del legislador no surtió efectos ya que los diputados de partido no fueron acreditados por riguroso orden, de acuerdo con el porcentaje de sufragios que hubieran logrado en relación a los demás candidatos del mismo partido, en todo el país.

Por otro lado, el artículo 134 de la Ley Electoral citada estableció que:

La calificación, cómputo y declaratoria correspondiente a las elecciones para senadores y diputados de mayoría y de partido, deberán realizarse con anterioridad a la fecha en que las respectivas Cámaras deben inaugurar su primer periodo ordinario de sesiones, por lo menos respecto del número de senadores y diputados indispensables para reunir el quórum reglamentario respectivo.

Las declaraciones correspondientes a las elecciones par Senadores y Diputados por mayoría, deberán hacerse antes del 15 de septiembre del año correspondiente al primer periodo ordinario de sesiones y las relati- 
vas a diputados de partido deberán quedar hechas a más tardar el día 30 del mismo mes.

Este artículo también resulta relevante, porque está vinculado al problema de la categoría de los diputados de partido. Ahora bien, según la fracción $\mathrm{V}$ del artículo 54 constitucional vigente en aquel momento: "Los diputados de mayoría y los de partido, siendo representantes de la Nación como lo establece el artículo 51, tendrán la misma categoría e iguales derechos y obligaciones".

No obstante esta aseveración, sí existía una diferencia que separaba a los diputados de partido de los de mayoría. Nos referimos a la que establecía el artículo 14 reformado, del Reglamento para el Gobierno Interior del Congreso General de los Estados Unidos Mexicanos, que consistía en que:

Si al llegar la fecha de apertura de sesiones de la Cámara de Diputados en el Primer Periodo Ordinario de su ejercicio, no han sido resueltos aun los casos de los diputados de partido, éstos no contarán para efecto del quórum necesario, bastando para legitimar la apertura de la Cámara con la asistencia de más de la mitad de los diputados de mayoría.

Por otro lado, el mismo artículo establecía que: "Resueltos que sean los casos de los diputados de partidos, el quórum se formará con la mitad más uno de todos los diputados en ejercicio".

Ahora bien, Mario Moya Palencia, escribió en su momento, respecto a la anterior diferencia, "que no establece restricción constitucional alguna, sino que solamente implica la solución del problema que puede presentarse en el estudio y resolución, por parte del Colegio Electoral, de los expedientes de partidos minoritarios con derecho a diputados de partido". ${ }^{6}$

Nosotros coincidimos parcialmente con esta opinión de quien fuera un destacado estudioso del derecho constitucional mexicano, porque consideramos que el artículo 14 del Reglamento para el Gobierno Interior del Congreso General en los años sesenta del siglo pasado, abría las puertas a una situación peligrosa que consiste en que como el PRI era el partido que obtenía la mayor parte de los diputados de mayoría, estaría en sus manos resolver sobre los diputados de partido, que eran los diputados de la oposición.

${ }^{6}$ Cfr. Moya Palencia, Mario, La reforma electoral, México, Plataforma, 1964, p. 157. 


\section{La disciplina partidaria}

Debido al históricamente célebre caso de los diputados rebeldes del PAN, quienes en 1958 como protesta por fraudes electorales no habían tomado posesión de su cargo, el artículo 150 estableció la suspensión de derechos políticos, hasta por seis años, a quienes habiendo sido electos diputados o senadores no se presentasen, sin causa justificada a juicio de la Cámara respectiva, a desempeñar el cargo dentro del plazo señalado en el primer párrafo del artículo 63 de la Constitución general de la República.

Por otro lado, el artículo 151 de la Ley Electoral estableció que:

La Secretaría de Gobernación cancelará temporal o definitivamente, previa garantía de audiencia, el registro de los Partidos Políticos Nacionales que habiendo postulado candidatos en una elección para diputados o senadores, acuerden que sus miembros que resulten electos no se presenten a desempeñar su encargo. Esta sanción se aplicará independientemente de la señalada en el artículo anterior.

La sanción de la cancelación temporal o definitiva, resaltaba la seriedad que debe tener el proceso electoral y obligaba a los partidos a canalizar sus inconformidades con el proceso electoral a través de los cauces legales que la ley establece y dentro de las reglas del juego del sistema de partidos regulado jurídicamente.

\section{Las reformas electorales de 1970 en México}

\section{El voto de los jóvenes}

La doctrina contemporánea del derecho electoral comparado establece que si bien es innegable la importancia de promover el voto entre los jóvenes, también es importante destacar que cualquier ejercicio de ingeniería legislativa respecto al tema de la edad mínima para votar debe tomar en cuenta los estudios que destacan grandes ventajas y desventajas de reducir la edad mínima para el ejercicio del voto activo.

Así, por un lado, la incorporación de una mayor cantidad de electores mediante la reducción de la edad mínima para ejercer el voto de 
entrada amplía el espectro ideológico y propositivo de un electorado, lo cual indiscutiblemente enriquece la vida política.

Asimismo, y como un efecto colateral, incorporar a los procesos electorales a mayor cantidad de jóvenes tiende a imbuirlos de un sentido de responsabilidad y de pertenencia institucional del que carecen si permanecen excluidos del proceso político de toma de decisiones.

Finalmente y sin que éstas ideas impliquen un listado exhaustivo de las ventajas de la reducción de la edad mínima para ejercitar el voto activo, la incorporación de los jóvenes a los procesos electorales desde temprana edad opera como un catalizador generalizado para sociedades con electorados adultos tradicionalmente apáticos, pasivos o poco participativos que reflejan un porcentaje de abstencionismo inconveniente. Esta última ventaja, se aprecia con claridad en 2012 en México con la irrupción de último momento en la escena política del movimiento juvenil "Yo soy 132".

Sin embargo, la otra cara de la moneda de las reformas legislativas que reduzcan la edad mínima para ejercer el voto activo serían las siguientes desventajas: en primer término y dependiendo del nivel cultural-educativo y de la solidez de las instituciones de educación primaria, secundaria y preparatoria, los jóvenes de 16 a 18 años tienden a ser fácilmente manipulables tratándose de temas económicos, financieros o constitucionales que implican per se una complejidad que supera tanto el nivel de escolaridad como la experiencia práctica de un individuo en el segmento poblacional de 16 a 18 años.

Esta supuesta proclividad a la manipulación de los jóvenes por parte de grupos o factores de poder avezados en la manipulación consciente o subliminal podría en determinados momentos y escenarios distorsionar los resultados de los procesos electorales mediante apoyos o animadversiones respecto a ciertos candidatos o partidos con decisiones tomadas quizá de manera visceral y no racional como conviene en el caso del ejercicio del voto activo. En el caso mexicano, por ejemplo, en pleno año 2012 y ante los resultados desafortunadamente negativos en las evaluaciones de maestros y profesores de todos los niveles escolares en nuestro país, cabría adelantar la conclusión de que un segmento importante del grupo poblacional de entre 16 a 18 años carecería de las herramientas teóricas y prácticas indispensables para ejercer un voto consciente e informado no por deficiencias personales sino por las carencias institucionales del sistema educativo nacional y debido a las inercias y resistencias sindicales y de factores y grupos de poder. 
Ahora bien, una vez consideradas de manera somera las ventajas y desventajas de reducir la edad mínima para ejercer el voto activo en México cabe destacar en un ejercicio comparativo internacional que en otras latitudes se han encontrado mecanismos intermedios sin llegar a una reducción general y nacional de la edad mínima citada mediante el establecimiento de modalidades interesantes como sería la reducción en el umbral mínimo en elecciones únicamente municipales $^{7}$ o bien en elecciones primarias internas de los partidos políticos como un campo de ensayo previo a una reforma constitucional que implicase la reducción de la edad mínima para votar en elecciones nacionales de gran trascendencia.

Asimismo, cabe destacar que incluso a nivel comparativo mundial no existe unanimidad con respecto a la conveniencia de reducir "en exceso" la edad mínima para ejercer el voto activo. En este sentido, por ejemplo, a nivel comparativo mundial la edad mínima para votar cubre un espectro que va desde los 16 y hasta los 21 años, y si bien la mayoría de los países se han inclinado ya por establecer los 18 años como la edad mínima ideal, existen países de innegable tradición cultural y desarrollo tecnológico como Japón que aún conservan como edad mínima para votar los 20 años. Incluso países como Arabia Saudita establecen una edad mínima de 21 años. Ahora bien, en el caso japonés, la explicación se encuentra parcialmente en el hecho de que la sociedad japonesa es conservadora y tradicionalista desde antaño y existe una valoración atípica y correcta desde nuestro punto de vista del valor de la experiencia y contribuciones de las personas de la tercera edad que no existe en algunas otras culturas o países del mundo y estas diferencias culturales explicarían el por qué un país tan avanzado cultural y tecnológicamente como Japón conserve una edad mínima para votar de 20 años bajo un criterio de que la toma de decisiones cruciales a través de elecciones en un país requiere de un mínimo de escolaridad y experiencia práctica de la vida, lo que probablemente haría inconveniente la reducción de la edad mínima para votar en el país nipón a 18 o 16 años. Recomendamos consultar el banco de datos y las gráficas y mapas con respecto a este tema que publica la Red de Cono-

7 How old is old enough? - the minimum age of voting and candidacy in UK elections: consultation paper, July 2003, UK Electoral Commission, para. 3.6. 
cimientos ACE en su página de Internet y que despliega la gama completa de edades mínimas para ejercer el voto activo a nivel mundial. ${ }^{8}$

Volviendo al caso mexicano, fue el 22 de diciembre de 1969, que se publicó en el Diario Oficial de la Federación, la enmienda constitucional al artículo 34 que reducía de 21 años a 18 años la edad requerida para que los mexicanos no casados gozaran plenamente de la ciudadanía.

Como consecuencia lógica de la anterior reforma, el 29 de enero de 1970, se publicaron las reformas a varios artículos de la Ley Electoral Federal de 1951, a fin de que la edad requerida para que un individuo disfrutara de derechos políticos activos fuese de 18 años cumplidos.

La citada reforma tuvo como sustento la idea - que ya adelantamos arriba con más detalle- de que en razón del dinamismo de los procesos de transformación social contemporáneos, los seres humanos evidencian un proceso de maduración más acelerado que los coloca en posición de responsabilidad a una edad más temprana que antaño, lo cual hacía anacrónica la disposición que establecía la edad de 21 años como el umbral para que un individuo soltero ejerciera el voto activo en un país, en el cual una porción importante de la población estaba ya - desde los años sesenta-compuesta por jóvenes.

Por otro lado, no se puede dejar de señalar que el seminal movimiento estudiantil de 1968 dejó claramente evidenciado el deseo y la necesidad impostergable de expresión política de la juventud de aquella época, por lo que indudablemente este factor debió estar presente en la mente de quienes impulsaron la citada reforma.

Finalmente, no podemos dejar de hacer una consideración de estadística electoral de gran relevancia. Gracias a que en México desde 1970 los jóvenes pueden ejercer el voto activo desde los 18 años, es que en la elección crucial presidencial de 2012 los ciudadanos de 18 y 19 años que suman 3418752 electores y que comprenden el $4.3 \%{ }^{9}$ del total de ciudadanos tendrán un peso determinante en una elección altamente competitiva, situación que no se presentaría para este segmento juvenil del electorado de 18 y 19 si la edad mínima para votar fuese de 20 años como lo es actualmente en Japón.

8 http://aceproject.org/epic-es/CDTable?question=VR001\&view=country\&set_ language $=$ es.

9 Gómez, Leslie, "Pueden los jóvenes definir las elecciones", Periódico Reforma, 28 de mayo de 2012, p. 8. 


\section{El sufragio por fórmulas}

Por otro lado, con la misma fecha de la reforma que otorgó el voto a los jóvenes, se estableció el registro mediante fórmula para los candidatos a diputados y senadores, quedando integrada cada fórmula por un candidato propietario y uno suplente. De esta manera, se llenó una laguna legal que había sido fuente de muchas disputas.

Por otra parte, se estableció que dentro del plazo del registro de candidatos, los partidos podrían sustituir libremente a los que ya hubieran registrado. Pero pasado el plazo, los partidos sólo podrían solicitar ante la Comisión Federal Electoral la cancelación del registro de uno o varios candidatos si la causa era fallecimiento, la inhabilitación o la incapacidad del candidato.

\section{Las reformas constitucionales de 1972 al sistema electoral}

\section{El derecho al voto pasivo de los jóvenes}

El 14 de febrero de 1972, se reformaron los artículos 55, fracción II, y 58 constitucionales, con lo que se redujo la edad para el ejercicio de los derechos políticos pasivos, esto es para ser votado.

En cuanto a las diputaciones se refiere, la edad mínima requerida al día de la elección se disminuyó de 25 a 21 años, y para poder ser senador de la República, se redujo de 35 a 30 años.

Cabe señalar que esta reforma fue la mancuerna faltante de la reforma constitucional que redujo la edad tratándose del voto activo, y que representó de manera más contundente la voluntad estatal de responder a la efervescencia política de la juventud mexicana secuela del movimiento de 1968, y que dio paso a la llamada "efebocracia", que es como se llamó popularmente a la andanada de jóvenes políticos que irrumpieron en el escenario nacional de esa época.

Por último, cabe señalar que las reformas que redujeron las edades mínimas para ejercer el voto activo y pasivo, son un ejemplo claro de ingeniería electoral "curativa", puesto que el Estado responde a posteriori a una presión social ya existente con una reforma jurídica que canalice institucionalmente dicha presión. 
En otros casos, se resaltarán ejemplos de lo que nosotros calificamos como ingeniería electoral "preventiva", cuando el Estado se adelanta a la acumulación de presión social y antes del surgimiento de los fenómenos sociales crea el marco jurídico que regulará dichos fenómenos. En ese sentido, la LXI Legislatura, de la Cámara de Diputados mexicana, por ejemplo, optó en el periodo que corrió de 2009 a 2012 en los temas de las candidaturas independientes, la revocación del mandato, la segunda vuelta electoral y la reelección legislativa por una postura que esquivó la oportunidad de aplicar una ingeniería electoral preventiva y optó por posponer la regulación detallada y de vanguardia de dichos temas.

\section{La reforma del sistema de diputados de partido}

Debido a que - como ya vimos - la raquítica oposición no lograba alcanzar el $2.5 \%$ de la votación nacional requerida para poder acreditar diputados de partido, y se habían hecho malabarismos de interpretación para conceder escaños al PPS y al PARM, con la misma fecha de la reforma constitucional anterior se redujo de $2.5 \%$ a $1.5 \%$ el porcentaje de la votación total en las elecciones exigido para que los partidos políticos tuvieran derecho a acreditar dichas diputaciones.

Por otro lado, se elevó el número máximo obtenible de diputados de partido de 20 a 25 por cada partido, y se establecieron las condiciones del acreditamiento, según los sufragios que hubiesen obtenido.

Los partidos de oposición tuvieron bajo la nueva reforma los siguientes resultados en 1973: el PAN obtuvo 25 curules, 4 de ellas de mayoría, el PPS 10 y el PARM 7; por otro lado, el PRI logró obtener 189 curules de mayoría.

\section{La Ley Federal Electoral de 1973}

El 5 de enero de 1973, fue expedida una nueva Ley Federal Electoral que siguió la tendencia de las anteriores leyes electorales mexicanas, de observar una "maximalización" progresiva en la regulación electoral, esto es, un incremento tanto en los aspectos de los procesos electorales que son regulados como en el número total de disposiciones 
o artículos jurídicos con los que se regulan los diversos temas electorales.

\section{Los partidos políticos}

La Ley Federal Electoral de 1973 "fue nutrida con todos los adelantos $\mathrm{y}$ aciertos de sus antecesoras, y fue elaborada con superior técnica jurídica y más depurada sistematización". ${ }^{10}$

Ahora bien, en cuanto a los partidos políticos, la ley de 1973 les dedicó 24 artículos, en los que reguló minuciosamente lo relativo a su concepto y fundamentos, constitución y registro, derechos y obligaciones, prerrogativas y propaganda electoral. A continuación, analizaremos las principales innovaciones de la citada ley.

\section{A. El concepto jurídico de partido político}

El artículo 17 de la Ley Federal Electoral de 1973 estableció que:

Los partidos políticos nacionales son asociaciones instituidas en los términos de esta ley, integradas por ciudadanos en pleno ejercicio de sus derechos políticos, para fines electorales, de educación cívica y orientación política.

Los partidos políticos concurren a la formación de la voluntad política del pueblo. Comparten en los organismos electorales la responsabilidad del proceso electoral y de vigilar que este se desarrolle conforme a los preceptos constitucionales y las disposiciones de esta ley.

Esta definición de qué es un partido político constituye un adelanto respecto al concepto contemplado en la anterior ley electoral, porque agrega el ingrediente de la educación cívica, al de orientación política, significando con esto, que se buscaba, así fuese incipientemente, formar una tradición política-electoral en el electorado mexicano. Asimismo, los partidos no eran considerados sólo meros auxiliares de los organismos electorales, sino que se les reconocía que concurrían a la formación de la voluntad política de la ciudadanía, compartiendo

${ }_{10}$ Cfr. López Moreno, Javier, op. cit., p. 74. 
con los organismos electorales la gran responsabilidad de velar por la limpieza del proceso electoral.

\section{B. Los estatutos}

El artículo 22 de la ley de 1973, en sus fracciones II y III, estableció como novedades, que los estatutos de los partidos políticos especificarían los procedimientos de afiliación y los derechos y obligaciones de sus miembros, así como también los sistemas de elección interna para la renovación de sus cuadros dirigentes que no podrían consistir en actos públicos semejantes a los comicios constitucionales.

Las anteriores disposiciones penetran en la vida interna de los partidos más profundamente que las anteriores leyes, puesto que exigen la publicidad de sus medios de afiliación y elección internas. Esta disposición, indudablemente, hubiera sido más democrática si se hubiera fijado, por ejemplo, que los partidos debían adoptar procedimientos de afiliación que respetasen la autonomía de la voluntad, prohibiendo afiliaciones colectivas realizadas sin el consentimiento del interesado, aunque es claro que para el contexto político mexicano de la época esto hubiera sido impensable.

\section{Los requisitos de constitución y registro de los partidos}

La ley de 1973 fijó en su artículo 23, los requisitos necesarios para que un partido político pudiese constituirse como tal y solicitar posteriormente su registro. Así, un partido debía:

I. Contar con un mínimo de dos mil afiliados en cada una, cuando menos, de las dos terceras partes de las entidades federativas, siempre que el número total de afiliados en todo el país no sea inferior a sesenta y cinco mil.

II. Haber celebrado cuando menos en cada una de las dos terceras partes de las entidades de la República, una asamblea en presencia de un juez, notario público o funcionario que haga sus veces, quien certificara:

1. Que fueron exhibidas listas nominales de afiliados de la entidad respectiva, clasificadas por municipios o delegaciones;

2. Que concurrieron al acto cuando menos los dos mil afiliados a que se refiere la fracción I y que comprobó con base en las listas nominales, la identidad y residencia de un cinco por ciento, cuando menos, del mínimo de afiliados requerido; 
3. Que entre los presentes se encontraban afiliados avecindados en, cuando menos, la mitad de los municipios o delegaciones de la entidad, en un mínimo de veinticinco personas por municipio o delegación;

4. Que fueron aprobados su declaración de principios, programa de acción y estatutos, $y$

5. Que se eligieron delegados propietarios y suplentes para la asamblea nacional constitutiva del partido en la forma prevista en sus estatutos.

III. Haber celebrado una asamblea nacional constitutiva ante la presencia de un notario público, quien certificara:

1. Que asistieron los delegados propietarios o suplentes elegidos en las asambleas locales y que acreditaron, por medio de los certificados correspondientes, que estas se celebraron de conformidad con lo prescrito en la fracción II;

2. Que comprobó la identidad y residencia de los delegados, por medio de la credencial permanente de elector y otro documento fehaciente, y

3. Que fueron aprobados su declaración de principios, programa de acción y estatutos.

Las actuaciones y documentos a que se refiere esta fracción debían quedar debidamente protocolizados.

Ahora bien, si por un lado se disminuyó el mínimo de afiliados de 75000 a 65000 , por otro lado, la exigencia de que los 2000 miembros de cada entidad estuvieran avecindados, cuando menos, en la mitad de los municipios o delegaciones de dicha entidad, con un mínimo de 25 personas por municipio o delegación, implicaba que, en realidad, bajo las nuevas disposiciones la constitución de un partido se volvía más difícil. ${ }^{11}$

Por otro lado, se extendió a 120 días naturales el plazo para la resolución de la Secretaría de Gobernación relativa a la solicitud de registro de un partido.

\section{Los derechos, obligaciones y prerrogativas de los partidos}

Bajo la Ley Federal Electoral de 1973, se estableció que los partidos políticos nacionales tenían el derecho y la obligación de integrarse a la Comisión Federal Electoral, a las comisiones locales y a los comités distritales electorales mediante un comisionado con voz y voto. Asi-

11 Ibidem, p. 76. 
mismo, los partidos tenían derecho a nombrar a un representante de casilla de los distritos en los que postulaban candidatos.

Por otro lado, las principales obligaciones de los partidos eran:

I. Observar las prescripciones consignadas en su declaración de principios y programa de acción;

II. Mantener el mínimo de afiliados en las entidades federativas y en todo el país, requerido para su constitución y registro;

III. Ostentarse con la denominación, emblema y color o colores que tengan registrados;

IV. Observar los procedimientos de afiliación, practicar los sistemas de elección interna de sus cuadros dirigentes y candidatos, así como a funcionar a través de sus órganos fundamentales, en los términos de esta ley, y

V. Mantener oficinas, editar una publicación propia por lo menos mensual y sostener centros de cultura cívica para sus miembros.

Ahora bien, sin duda una de las principales innovaciones de la ley de 1973, fue el establecimiento de un capítulo de prerrogativas de los partidos políticos, que a las exenciones fiscales - ya conocidas- añadió las franquicias postales y telegráficas necesarias para el cumplimiento de sus funciones.

Asimismo, el artículo 39 de la ley de 1973 permitió el acceso a la radio y televisión a los partidos, aunque sólo durante los periodos de campana electoral. No obstante "que el otorgamiento de los tiempos precisos en ambos medios y la producción de los programas"12 eran hechos por la Secretaría de Gobernación, es innegable que esta prerrogativa significó un avance considerable en la reglamentación jurídica de los partidos, y en su posibilidad de hacer llegar a la ciudadanía sus mensajes y posturas políticas.

Por otro lado, y con una visión retrospectiva - lo que indudablemente facilita nuestro análisis- desde la óptica del año 2012, es fácil apreciar que quizá es menos pernicioso un sistema de acceso a los medios electrónicos tradicionales controlado incluso hasta por la Secretaría de Gobernación que uno como el que se abandonó - afortunadamente- con la reforma electoral de 2007, en el que el duopolio televisivo mexicano aprovechaba el esquema establecido para su be-

12 Cfr. Paoli, op. cit., p. 203. 
neficio económico y lo que es aún más grave para incidir en la propia contienda electoral.

En el anterior sentido, se observa en este ejemplo con meridiana claridad que la evolución de la normativa electoral en ocasiones nos permite apreciar titubeos en el diseño de modelos de comunicación político-electorales inevitables debido a que las ventajas o desventajas de un determinado sistema de acceso de los partidos políticos y candidatos a los medios sólo se aprecian a posteriori con la implementación práctica de un modelo de comunicación política determinado.

Así, por ejemplo, un modelo de "libre contratación" directa de tiempos y espacios publicitarios entre los actores políticos y las empresas televisoras desencadenó en México una gran cantidad de problemas y conflictos sistémicos inexistentes bajo sistemas aparentemente más arcaicos y con la presencia ya sea del Estado mismo o de los órganos electorales. Ahora bien, aunque los modelos "sin libertad de contratación”: el de la participación estatal vía la Secretaría de Gobernación, por un lado, o el del control del tema por parte del Consejo General del IFE por el otro, implican riesgos de intervención gubernamental a favor del partido político en el poder, éstos resultan quizá más equitativos porque al existir representación ciudadana y partidaria en dichos órganos de control - tanto en el manejo por parte del Estado como en el de la competencia exclusiva del Consejo General del IFE- es menos probable que factores de poder no ciudadanos o no supervisados por los propios representantes de los partidos y sí vinculados al poder mediático irrumpan descaradamente y sin control en la contratación, producción y transmisión de contenidos político-electorales, lo que siempre inclina la balanza en contra de modelos de libre mercado sin participación o intervención estatal o bien de los órganos electorales y a favor de esquemas con contrapesos, controles, candados y presencia ciudadana y/o partidista en los órganos de decisión en la asignación distribución y difusión de espacios electorales.

\section{El Registro Nacional de Electores}

En lo que concierne al Registro Nacional de Electores, se estableció su autonomía administrativa, así como la obligación de dar a los partidos 
políticos las listas nominales de electores y la facultad de elaborar las estadísticas electorales. ${ }^{13}$

\section{Demarcaciones electorales}

Respecto de la configuración de la geografía electoral, la ley de 1973 estableció como innovación la división seccional como la demarcación territorial electoral en que se dividen los distritos para la recepción del sufragio y con base en la cual se formulan las listas de electores. Cada sección comprendió un máximo de 3000 electores y un mínimo de 100 y tantas casillas como determinara el Comité Distrital. ${ }^{14}$

Bajo el esquema electoral de la ley de 1973, en las elecciones de 1976, el PAN obtuvo veinte escaños, el PPS doce y el PARM diez.

Por otro lado, en las elecciones presidenciales de 1976, el panorama fue quizá el más desalentador de la oposición en casi 50 años.

El candidato del PRI, José López Portillo, obtuvo el 98.18\% de los votos, siendo el único candidato, puesto que el PAN no presentó candidato y tanto el PPS como el PARM apoyaron la candidatura de López Portillo.

Bajo este panorama político desolador, fue que el gobierno de José López Portillo concibió la reforma política de 1977, que vista en retrospectiva fincó las bases del sofisticado andamiaje electoral de vanguardia con el que contamos los mexicanos en pleno año 2012 y como consecuencia de una paciente y costosísima labor colectiva de miles de ciudadanos empeñados en construir un sistema político democrático duradero que tenemos continuamente que revalorar y apreciar recordando las etapas iniciales del reconocimiento, regulación jurídica y constitucionalización de los partidos políticos como entidades indispensables para la estabilidad política nacional.

13 Cfr. López Moreno, Javier, op. cit., p. 77.

${ }^{14}$ Cfr. González Casanova, Pablo, El Estado y los partidos políticos en México, México, Era, 1981, p. 67. 